\title{
Acoustic interaction forces between small particles in an ideal fluid
}

\author{
Silva, Glauber T.; Bruus, Henrik
}

Published in:

Physical Review E

Link to article, DOI:

10.1103/PhysRevE.90.063007

Publication date:

2014

Document Version

Publisher's PDF, also known as Version of record

Link back to DTU Orbit

Citation (APA):

Silva, G. T., \& Bruus, H. (2014). Acoustic interaction forces between small particles in an ideal fluid. Physical Review E, 90(6), 063007. https://doi.org/10.1103/PhysRevE.90.063007

\section{General rights}

Copyright and moral rights for the publications made accessible in the public portal are retained by the authors and/or other copyright owners and it is a condition of accessing publications that users recognise and abide by the legal requirements associated with these rights.

- Users may download and print one copy of any publication from the public portal for the purpose of private study or research.

- You may not further distribute the material or use it for any profit-making activity or commercial gain

- You may freely distribute the URL identifying the publication in the public portal

If you believe that this document breaches copyright please contact us providing details, and we will remove access to the work immediately and investigate your claim 


\title{
Acoustic interaction forces between small particles in an ideal fluid
}

\author{
Glauber T. Silva ${ }^{1, *}$ and Henrik Bruus ${ }^{2, \dagger}$ \\ ${ }^{1}$ Physical Acoustics Group, Instituto de Física, Universidade Federal de Alagoas, Maceió, Alagoas 57072-970, Brazil \\ ${ }^{2}$ Department of Physics, Technical University of Denmark, DTU Physics Building 309, DK-2800 Kongens Lyngby, Denmark
}

(Received 24 August 2014; published 9 December 2014)

\begin{abstract}
We present a theoretical expression for the acoustic interaction force between small spherical particles suspended in an ideal fluid exposed to an external acoustic wave. The acoustic interaction force is the part of the acoustic radiation force on one given particle involving the scattered waves from the other particles. The particles, either compressible liquid droplets or elastic microspheres, are considered to be much smaller than the acoustic wavelength. In this so-called Rayleigh limit, the acoustic interaction forces between the particles are well approximated by gradients of pair-interaction potentials with no restriction on the interparticle distance. The theory is applied to studies of the acoustic interaction force on a particle suspension in either standing or traveling plane waves. The results show aggregation regions along the wave propagation direction, while particles may attract or repel each other in the transverse direction. In addition, a mean-field approximation is developed to describe the acoustic interaction force in an emulsion of oil droplets in water.
\end{abstract}

DOI: 10.1103/PhysRevE.90.063007

PACS number(s): 47.35.Rs, 43.25.Qp, 43.25.+y, 47.15.-x

\section{INTRODUCTION}

Techniques relying on acoustofluidic forces, such as acoustic radiation force and streaming, are currently used in many different ways to handle suspended cells, microparticles, and fluids nonintrusively and label free in microfluidic setups such as separation, trapping, and sorting of cells, particle manipulation, as well as generation and control of fluid motion [1-3]. Experimentally, ultrasound waves emitted into a particle suspension give rise to acoustic streaming of the carrier fluid [4], and they are responsible for the two acoustofluidic forces driving the acoustophoretic motion of the suspended particles: the acoustic radiation force and the Stokes drag force from acoustic streaming. The theoretical description of these complex, nonlinear acoustic effects is not yet complete, and in this paper we develop the theory of the acoustic radiation force, which dominates the motion of the larger microparticles [5].

Concerning the radiation force exerted on a single particle, the so-called primary radiation force $\boldsymbol{F}^{\mathrm{rad}}$, recent studies by Doinikov [6] and Danilov and Mironov [7], as well as Settnes and Bruus [8] and Silva [9], have advanced the theoretical treatment beyond the seminal contributions by King [10], Yosioka and Kawasima [11], and Gorkov [12]. The main improvement found in these recent studies is the introduction of thermoviscous effects in both the incident ultrasound waves and the scattered wave from the particle. However, in a particle suspension exposed to an external acoustic wave, a secondary radiation force appears, the so-called acoustic interaction force $\boldsymbol{F}_{\text {int }}^{\text {rad }}$. For a given particle, the acoustic interaction force is caused by the scattered waves from the other particles. Investigations on this force dates back to the 19th century, when Bjerknes studied the mutual force between a pair of bubbles [13], and the analysis performed by König on the acoustic interaction force between two rigid spheres [14]. Subsequently, this force was investigated considering

\footnotetext{
*glauber@pq.cnpq.br

†bruus@fysik.dtu.dk
}

short-range interaction between particles of the types rigidrigid [15,16], bubble-bubble [17,18], bubble-rigid [19], and bubble-droplet [20], whereas long-range rigid-rigid [21] and bubble-bubble [22,23] interactions have also been studied. The acoustic interaction force between two droplets aligned relative to an incident plane wave with arbitrary interparticle distance was also analyzed [24]. Moreover, bubble-bubble interaction at any separation distance has also been analyzed through a seminumerical scheme based on the partial-wave expansion method and the translational addition theorem of spherical functions [25].

The current literature on the acoustic interaction force lacks an investigation on a suspension composed of compressional fluid droplets or solid elastic particles without any restriction on the interparticle distances. These kinds of particles are often used in experiments on acoustofluidics, acoustical tweezers, and demulsification of particle-water mixtures by ultrasound. It is our goal here to provide an analytical expression for the acoustic interaction force between suspended droplets or solid elastic microparticles in an inviscid fluid. The proposed method, which takes the form of a scalar potential theory for the acoustic interaction force, extends the single-particle radiation force theory developed by Gorkov [12] to include rescattering events between particles in the suspension. The method is applied to various examples of the acoustic interaction force in the case of either a standing or a traveling external plane wave, and a mean-field theory is proposed and applied to compute the acoustic interaction force between the drops in an emulsion of oil drops in water.

\section{THEORY}

The linear wave theory for the acoustic fields in an unbounded, isotropic fluid of density $\rho_{0}$ and isentropic compressibility $\kappa_{0}=1 /\left(\rho_{0} c_{0}^{2}\right)$, where $c_{0}$ is the adiabatic sound velocity in the fluid, is standard textbook material [26-28]. We neglect the viscous dissipation of the acoustic field in the particle suspension, which is a good approximation for particle radii much larger than the width of the viscous 
boundary layer [8] and for frequencies much lower than hypersound frequencies (below $\mathrm{GHz}$ for water). Consequently, a time-harmonic acoustic wave can be described by the velocity potential $\Phi(\boldsymbol{r}, t)$, where $\boldsymbol{r}$ is position and $t$ is time, in terms of a complex-valued phase factor $e^{-i \omega t}$, where $\omega$ is the angular wave frequency, and an amplitude function $\phi(\boldsymbol{r})$, which satisfies the Helmholtz wave equation,

$$
\begin{aligned}
\Phi(\boldsymbol{r}, t) & =\phi(\boldsymbol{r}) e^{-i \omega t}, \\
\nabla^{2} \phi(\boldsymbol{r}) & =-k^{2} \phi(\boldsymbol{r}), \text { with } k=\frac{\omega}{c_{0}} .
\end{aligned}
$$

In terms of the potential $\phi(\boldsymbol{r})$, the amplitude function of the pressure $p(\boldsymbol{r})$, the density $\rho(\boldsymbol{r})$, and the velocity $\boldsymbol{v}(\boldsymbol{r})$ are given by

$$
\begin{aligned}
& p(\boldsymbol{r})=i \omega \rho_{0} \phi(\boldsymbol{r}), \\
& \rho(\boldsymbol{r})=i \frac{\omega \rho_{0}}{c_{0}^{2}} \phi(\boldsymbol{r}), \\
& \boldsymbol{v}(\boldsymbol{r})=\nabla \phi(\boldsymbol{r}) .
\end{aligned}
$$

In the following we outline some fundamental concepts of acoustic scattering and radiation forces on small particles suspended in the fluid.

\section{A. Single-particle scattering in the Rayleigh limit}

Consider a monochromatic acoustic wave represented by the velocity potential amplitude $\phi_{\text {in }}(\boldsymbol{r})$ incident on and scattering off a small spherical particle suspended in the medium. The scattered wave adds to the acoustic wave incident on any other particle in the suspension, so the first particle acts as a source of additional acoustic radiation forces felt by the other particles in the suspension. All physical quantities related to this source particle are marked by the subscript " $s$ " such as particle radius $a_{s}$, density $\rho_{s}$, isentropic compressibility $\kappa_{s}$, and center position $\boldsymbol{r}_{s}$, as sketched in Fig. 1. At any given probe position $\boldsymbol{r}_{p}$, the outgoing scattered wave from the source particle is represented by the velocity potential

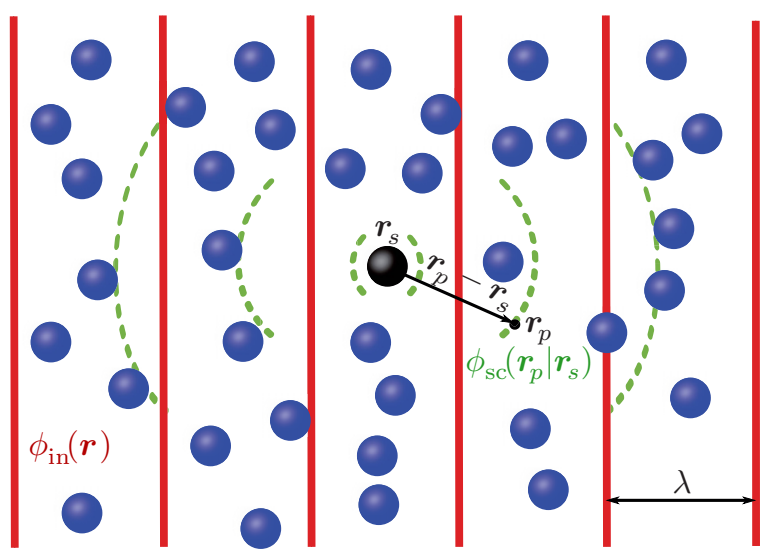

FIG. 1. (Color online) Sketch of the external incident wave $\phi_{\text {in }}(\boldsymbol{r})$ (red straight lines) scattered by a suspension of small spherical particles with radii $a_{s} \ll \lambda$. The scattered wave $\phi_{\mathrm{sc}}\left(\boldsymbol{r}_{p} \mid \boldsymbol{r}_{s}\right)$ (green dashed curves) from a source particle located at $\boldsymbol{r}_{s}$ (black sphere) is probed at the position $\boldsymbol{r}_{p}$. amplitude function $\phi_{\mathrm{sc}}\left(\boldsymbol{r}_{p} \mid \boldsymbol{r}_{s}\right)$, where subscript " $p$ " here and in the following relates to the probe. Throughout this work, we only consider the so-called Rayleigh scattering limit $k a_{s} \ll 1$. We also assume ideal scattering boundary conditions, i.e., total absorption without reflection of any scattered waves at infinity. In this limit, the acoustic scattering is dominated by the monopole and dipole scattering, and the scattered wave is given by [29]

$$
\begin{aligned}
\phi_{\mathrm{sc}}\left(\boldsymbol{r}_{p} \mid \boldsymbol{r}_{s}\right)= & i f_{0, s} \frac{a_{s}^{3} \omega}{3 \rho_{0}} \frac{\rho_{\mathrm{in}}\left(\boldsymbol{r}_{s}\right) e^{i k R_{p s}}}{R_{p s}} \\
& -f_{1, s} \frac{a_{s}^{3}}{2} \nabla_{p} \cdot\left[\frac{\boldsymbol{v}_{\mathrm{in}}\left(\boldsymbol{r}_{s}\right) e^{i k R_{p s}}}{R_{p s}}\right]+O\left[\frac{\left(k a_{s}\right)^{5}}{\left(k R_{p s}\right)^{3}}\right],
\end{aligned}
$$

where $R_{p s}=\left|\boldsymbol{r}_{p}-\boldsymbol{r}_{s}\right|, \nabla_{p}$ is nabla acting on $\boldsymbol{r}_{p}$, and terms of the order $\left(k a_{s}\right)^{5} /\left(k R_{p s}\right)^{3}$ arise from the quadrupolar scattering [30]. The monopole and dipole scattering factors $f_{0, s}$ and $f_{1, s}$ of the source particle are given in terms of the density ratio $\tilde{\rho}_{s}=\rho_{s} / \rho_{0}$ and the compressibility ratio $\tilde{\kappa}_{s}=\kappa_{s} / \kappa_{0}$ as follows $[11,12]:$

$$
\begin{aligned}
& f_{0, s}=1-\tilde{\kappa}_{s}, \\
& f_{1, s}=\frac{2\left(\tilde{\rho}_{s}-1\right)}{2 \tilde{\rho}_{s}+1} .
\end{aligned}
$$

For the analysis of the higher-order scattering, it is useful to introduce the scattering parameters $\epsilon_{s}$ and $\epsilon_{p}$, as well as the dimensionless probe-source distance $x_{p s}$,

$$
\epsilon_{s}=k a_{s}, \quad \epsilon_{p}=k a_{p}, \quad x_{p s}=k R_{p s} .
$$

This, together with Eqs. (2b) and (2c), can be used to rewrite Eq. (3) in terms of a scattering operator acting on the incident wave $\phi_{\text {in }}\left(\boldsymbol{r}_{S}\right)$ as

$$
\begin{aligned}
\phi_{\mathrm{sc}}\left(\boldsymbol{r}_{p} \mid \boldsymbol{r}_{s}\right)= & -\epsilon_{s}^{3} \frac{e^{i x_{p s}}}{x_{p s}}\left[\frac{f_{0, s}}{3}+\frac{i f_{1, s}}{2}\left(1+\frac{i}{x_{p s}}\right) \partial_{x_{p s}}\right] \phi_{\mathrm{in}}\left(\boldsymbol{r}_{s}\right) \\
& +O\left({\tilde{\epsilon_{s}}}^{5}\right)
\end{aligned}
$$

where $\tilde{\epsilon}_{s}=\epsilon_{s} / x_{p s}^{3 / 5}$. Note that $R_{p s} \sim a_{s}$ implies $x_{p s} \sim \epsilon_{s}$, and thus $\phi_{\mathrm{sc}}\left(\boldsymbol{r}_{p} \mid \boldsymbol{r}_{s}\right)=O\left(\epsilon_{s}\right)$ for probes near the source.

\section{B. Single-particle radiation force}

Once the scattering velocity potential in Eq. (6) is known, the resulting acoustic radiation force acting on a suspended probe particle of radius $a_{p}$ and scattering coefficients $f_{0, p}$ and $f_{1, p}$ placed at $\boldsymbol{r}_{p}$ can be calculated in standard manners using second-order time-averaged perturbation theory in the pressure or the particle velocity amplitude $[8,11,12]$. In the Rayleigh scattering limit for any incident acoustic wave $\phi_{\text {in }}(\boldsymbol{r})$, except plane traveling waves, the radiation force $\boldsymbol{F}^{\mathrm{rad}}\left(\boldsymbol{r}_{p}\right)$ is a gradient of a potential $U$ given by

$$
\begin{aligned}
\boldsymbol{F}^{\mathrm{rad}}\left(\boldsymbol{r}_{p}\right)= & -\nabla_{p} U\left(\boldsymbol{r}_{p}\right), \\
U\left(\boldsymbol{r}_{p}\right)= & -\frac{\epsilon_{p}^{3} \pi \rho_{0}}{k}\left[\frac{f_{0, p}}{3}\left|\phi_{\mathrm{in}}\left(\boldsymbol{r}_{p}\right)\right|^{2}-\frac{f_{1, p}}{2}\left|\tilde{\nabla}_{p} \phi_{\mathrm{in}}\left(\boldsymbol{r}_{p}\right)\right|^{2}\right] \\
& +O\left(\tilde{\epsilon}_{p}^{5}\right),
\end{aligned}
$$


where $\tilde{\nabla}_{p}=(1 / k) \nabla_{p}$, used in Eq. (7b), is the dimensionless nabla operator convenient to use when calculating derivatives of the velocity potential.

\section{Scattering in a suspension of particles}

We now consider a specific configuration $\mathcal{S}$ of $N$ spherical particles arbitrarily placed at the positions $\boldsymbol{r}_{s}$ for $s=$ $1,2,3, \ldots, N$. The particle at position $\boldsymbol{r}_{s}$ has the monopole and dipole scattering coefficients $f_{0, s}$ and $f_{1, s}$, respectively, as well as radius $a_{s}$. All particles are assumed to have expansion parameters $\epsilon_{s}=k a_{s} \ll 1$.

An external incident wave with velocity potential $\phi_{\text {ext }}$ hits the $N$-particle suspension and multiple-scattering processes occurs. The resulting acoustic field $\phi_{\text {in }}\left(\boldsymbol{r}_{p}\right)$ incident at the probe position $\boldsymbol{r}_{p}$ can thus be written as

$$
\phi_{\mathrm{in}}\left(\boldsymbol{r}_{p}\right)=\phi_{\mathrm{ext}}\left(\boldsymbol{r}_{p}\right)+\phi_{\mathrm{msc}}\left(\boldsymbol{r}_{p} \mid \mathcal{S}\right)
$$

where $\phi_{\mathrm{msc}}\left(\boldsymbol{r}_{p} \mid \mathcal{S}\right)$ is that part of the acoustic field at position $\boldsymbol{r}_{p}$ that is caused by prior multiscattering events at one or more particles in the configuration $\mathcal{S}$.

In the Rayleigh limit, the multiscattering contribution to the acoustic wave $\phi_{\text {in }}\left(\boldsymbol{r}_{p}\right)$ incident at the probe point $\boldsymbol{r}_{p}$ is dominated by scattering waves having undergone only a single prior scattering event at some source point $\boldsymbol{r}_{s}$ different from $\boldsymbol{r}_{p}$, written as $s \neq p$. To lowest scattering order, the multiple-scattering part $\phi_{\mathrm{msc}}\left(\boldsymbol{r}_{p} \mid \mathcal{S}\right)$ to the incident wave at $\boldsymbol{r}_{p}$ can thus be written as a sum over nonidentical pairs of positions,

$$
\phi_{\mathrm{msc}}\left(\boldsymbol{r}_{p} \mid \mathcal{S}\right)=\sum_{\boldsymbol{r}_{s} \in \mathcal{S}}^{\prime} \phi_{\mathrm{sc}}\left(\boldsymbol{r}_{p} \mid \boldsymbol{r}_{s}\right)+O\left(\tilde{\epsilon}^{6}\right)
$$

Here the primed summation means that the sum is performed in all suspended particles except $s=p$, and the expansion parameter is $\tilde{\epsilon}=\max _{s}\left\{\tilde{\epsilon}_{s}\right\}$.

\section{The acoustic interaction force}

When the particle interaction is taken into account through the scattered waves, the radiation force can be written as the sum of contributions from the unperturbed external field $\phi_{\text {ext }}\left(\boldsymbol{r}_{p}\right)$ and from the configuration-dependent interaction field, which involves terms like $\phi_{\text {ext }}^{*}\left(\boldsymbol{r}_{p}\right) \phi_{\mathrm{msc}}\left(\boldsymbol{r}_{p} \mid \mathcal{S}\right)$. By substituting Eq. (8) into Eq. (7b) we find

$$
\boldsymbol{F}^{\mathrm{rad}}\left(\boldsymbol{r}_{p}\right)=\boldsymbol{F}_{\mathrm{ext}}^{\mathrm{rad}}\left(\boldsymbol{r}_{p}\right)+\boldsymbol{F}_{\mathrm{int}}^{\mathrm{rad}}\left(\boldsymbol{r}_{p} \mid \mathcal{S}\right) .
$$

The radiation force $\boldsymbol{F}_{\mathrm{ext}}^{\mathrm{rad}}\left(\boldsymbol{r}_{p}\right)$ from the external field corresponds to $\phi_{\text {in }}=\phi_{\text {ext }}$ in Eq. (7b),

$$
\begin{aligned}
\boldsymbol{F}_{\mathrm{ext}}^{\mathrm{rad}}\left(\boldsymbol{r}_{p}\right)= & -\epsilon_{p}^{3} \pi \rho_{0} \tilde{\nabla}_{p}\left[\frac{f_{0, p}}{3}\left|\phi_{\mathrm{ext}}\left(\boldsymbol{r}_{p}\right)\right|^{2}-\frac{f_{1, p}}{2}\left|\tilde{\nabla}_{p} \phi_{\mathrm{ext}}\left(\boldsymbol{r}_{p}\right)\right|^{2}\right] \\
& +O\left(\tilde{\epsilon}_{p}^{5}\right) .
\end{aligned}
$$

It follows that the configuration-dependent acoustic interaction force can be expressed as a gradient force,

$$
\boldsymbol{F}_{\text {int }}^{\mathrm{rad}}\left(\boldsymbol{r}_{p} \mid \mathcal{S}\right)=-\nabla_{p} \sum^{\prime} U\left(\boldsymbol{r}_{p} \mid \boldsymbol{r}_{s}\right)+O\left(\tilde{\epsilon}_{p}^{5}\right) .
$$

For given probe and source positions $\boldsymbol{r}_{p}$ and $\boldsymbol{r}_{s}$ the pairinteraction potential energy is

$$
\begin{aligned}
U\left(\boldsymbol{r}_{p} \mid \boldsymbol{r}_{s}\right)= & \frac{\pi \epsilon_{p}^{3} \rho_{0}}{k} \operatorname{Re}\left[\frac{2 f_{0, p}}{3} \phi_{\mathrm{ext}}^{*}\left(\boldsymbol{r}_{p}\right) \phi_{\mathrm{sc}}\left(\boldsymbol{r}_{p} \mid \boldsymbol{r}_{s}\right)\right. \\
& \left.-f_{1, p} \tilde{\nabla}_{p} \phi_{\mathrm{ext}}^{*}\left(\boldsymbol{r}_{p}\right) \cdot \tilde{\nabla}_{p} \phi_{\mathrm{sc}}\left(\boldsymbol{r}_{p} \mid \boldsymbol{r}_{s}\right)\right] .
\end{aligned}
$$

The potential energy depends on a particle volume product $a_{p}^{3} a_{s}^{3}$ and on scattering factors like $f_{i, p} f_{i, s}$, with $i=0,1$. It is clear that the acoustic interaction force has the same dependence on these parameters. Note further that the potential energy $U\left(\boldsymbol{r}_{p} \mid \boldsymbol{r}_{s}\right)$ is not necessarily symmetric with respect to its indices. Thus, the acoustic interaction force may not be symmetric either.

We now move on to analyze to which order in $\epsilon_{p}$ the acoustic interaction force contributes to the total radiation force. To ensure consistent approximations, this contribution must appear with a smaller order in $\epsilon_{p}$ than the quadrupole $\tilde{\epsilon}_{p}^{5}$ contribution given in Eq. (7b). The pair-interaction approximation is more dominant when the dimensionless probe-source distance $x_{p s}$ is small, satisfying $k\left(a_{p}+a_{s}\right) \leqslant x_{p s}<1$. Combining Eqs. (11) and (10c), one can show that the leading contribution to the interaction force is

$$
\left|\boldsymbol{F}_{\text {int }}^{\mathrm{rad}}\right| \sim\left|\left[\tilde{\nabla}_{p} \phi_{\mathrm{ext}}^{*}\left(\boldsymbol{r}_{p}\right) \cdot \tilde{\nabla}_{p}\right] \tilde{\nabla}_{p} \phi_{\mathrm{sc}}\left(\boldsymbol{r}_{p} \mid \boldsymbol{r}_{s}\right)\right| \sim \frac{\epsilon_{p}^{3}}{x_{p s}^{4}} .
$$

We first note the strong suppression of $\boldsymbol{F}_{\text {int }}^{\mathrm{rad}}$ by $x_{p s}^{-4}$ as the particle distance increases above a few wavelengths, $x_{p s} \gtrsim 1$. Then we may express $x_{p s}$ in terms of the scattering parameter of the probe particle as $x_{p s}=\gamma \epsilon_{p}$, where $\gamma>1+a_{s} / a_{p}$. Therefore, $\left|\boldsymbol{F}_{\text {int }}^{\mathrm{rad}}\right|=O\left[\gamma^{-4} \epsilon_{p}^{-1}\right]$. Comparing the leading term in the acoustic interaction force with the quadrupole correction in Eq. (7b), we find that consistent approximations are obtained, given that $\gamma^{-4} \epsilon_{p}^{-1} \gg \tilde{\epsilon}_{p}^{5}$ or that $\gamma$ is restricted to the limited range $1+a_{s} / a_{p}<\gamma \ll \epsilon_{p}^{-3}$. For example, if $\epsilon_{p}=$ 0.1 , then $\gamma \ll 1000$; otherwise, the acoustic interaction force magnitude becomes comparable to the quadrupole correction, which was already neglected in the radiation force expression given in Eq. (7a).

\section{EXAMPLES OF THE ACOUSTIC PAIR-INTERACTION FORCE}

The acoustic interaction force exerted on a probe by a single source particle will be determined considering the interaction potential energy in Eq. (11) for an external traveling plane and standing wave. The source particle is at the origin of the coordinate system $\boldsymbol{r}_{s}=\mathbf{0}$, while the probe particle is at any other position $\boldsymbol{r}_{p}=\boldsymbol{r}=r \boldsymbol{e}_{r}$. Furthermore, the shorthand notation $U(\boldsymbol{r})=U(\boldsymbol{r} \mid \mathbf{0})$ and $\boldsymbol{F}_{\text {int }}^{\mathrm{rad}}(\boldsymbol{r})=\boldsymbol{F}_{\text {int }}^{\mathrm{rad}}(\boldsymbol{r} \mid \mathbf{0})$ will be used. 


\section{A. Traveling plane wave}

Consider an external plane wave propagating along the $z$ axis. The velocity potential amplitude of this wave is

$$
\phi_{\mathrm{ext}}(z)=\frac{v_{0}}{k} e^{i k z}
$$

where $v_{0}$ is the magnitude of the oscillatory velocity.

The pair-interaction potential energy is calculated by substituting Eq. (13) into Eq. (6). Thus, inserting the obtained result into Eq. (11), we find in spherical coordinates $(r, \theta, \varphi)$ that

$$
\begin{aligned}
U(r, \theta)= & \pi E_{0} k^{3} a_{p}^{3} a_{s}^{3}\left(\operatorname { c o s } [ k r ( 1 - \operatorname { c o s } \theta ) ] \left\{f_{1, p} f_{1, s} \frac{1+3 \cos 2 \theta}{2} \frac{1}{(k r)^{3}}\right.\right. \\
& \left.-\left[\frac{4}{9} f_{0, p} f_{0, s}+f_{1, p} f_{1, s} \frac{1+\cos 2 \theta}{2}-\frac{2}{3}\left(f_{0, s} f_{1, p}+f_{0, p} f_{1, s}\right) \cos \theta\right] \frac{1}{k r}\right\} \\
& \left.-\sin [k r(1-\cos \theta)]\left[\frac{1}{2} f_{1, p} f_{1, s}-\frac{2}{3}\left(f_{0, s} f_{1, p}+f_{0, p} f_{1, s}\right) \cos \theta+\frac{3}{2} f_{1, p} f_{1, s} \cos 2 \theta\right] \frac{1}{(k r)^{2}}\right),
\end{aligned}
$$

where $E_{0}=\frac{1}{2} \rho_{0} v_{0}^{2}$ is the characteristic energy density of the external traveling plane wave. Below we study two special cases of this expression, and in this context it is useful to introduce the compression and density interaction potential strengths, $U_{0}$ and $U_{1}$, respectively,

$$
\begin{aligned}
& U_{0}=\frac{4 \pi}{9} E_{0} k^{3} a_{p}^{3} a_{s}^{3} f_{0, p} f_{0, s}, \\
& U_{1}=\pi E_{0} k^{3} a_{p}^{3} a_{s}^{3} f_{1, p} f_{1, s} .
\end{aligned}
$$

As the first special case, we reproduce the seminal result for the secondary Bjerknes force between two bubbles, for which it is assumed that the external wave frequency is much smaller than the resonance frequency of the bubbles. Since for gas bubbles $f_{0} \approx-10^{5}$ and $f_{1} \approx-2$, and because $k r>k a_{s} \approx 10^{-3}$ implies that $f_{0} \gg f_{1} /(k r)$, only the term involving $f_{0, p} f_{0, s}$ is relevant in Eq. (14), and we arrive at at $U$ and $\boldsymbol{F}_{\text {int }}^{\text {rad }}=-\nabla U$,

$$
\begin{aligned}
U(r, \theta) & =-U_{0} \frac{\cos [k r(1-\cos \theta)]}{k r}, \\
\boldsymbol{F}_{\text {int }}^{\mathrm{rad}}(r, \theta) & =-k U_{0}\left\{\frac{\sin [k r(1-\cos \theta)] \sin \theta}{k r} \boldsymbol{e}_{\theta}+\frac{\cos [k r(1-\cos \theta)]+k r \sin [k r(1-\cos \theta)](1-\cos \theta)}{(k r)^{2}} \boldsymbol{e}_{r}\right\} \\
& \approx-\frac{k U_{0}}{(k r)^{2}} \boldsymbol{e}_{r}=-\frac{4 \pi E_{0} k^{2} a_{p}^{3} a_{s}^{3} \kappa_{p} \kappa_{s}}{9 \kappa_{0}^{2} r^{2}} \boldsymbol{e}_{r}, \quad k r \ll 1,
\end{aligned}
$$

where the latter is the secondary Bjerknes force in the short-range limit as derived by Zheng and Apfel [24].

As the second special case, we consider the acoustic interaction between particles collected in the transverse plane $(\theta=\pi / 2)$. Since the phase of the external wave does not change in the transverse plane, the angular dependence drops out of Eq. (14). In this special case, only the radial distance $\varrho=\sqrt{x^{2}+y^{2}}$ in the transverse plane and the associated in-plane radial unit vector $\boldsymbol{e}_{\varrho}$ play a role in the following. The potential $U$ becomes

$$
U(\varrho)=U_{0} n_{0}(k \varrho)+U_{1} \frac{n_{1}(k \varrho)}{k \varrho},
$$

where, respectively, the functions $n_{0}(x)=-\cos (x) / x$ and $n_{1}(x)=-\sin (x) / x-\cos (x) / x^{2}$ are the zero- and first-order spherical Neumann functions. In the short-range limit $k \varrho \ll 1$, the negative gradient of Eq. 17(a) gives

$$
\boldsymbol{F}_{\text {int }}^{\mathrm{rad}}(\varrho)=-k U_{1}\left[\frac{3}{(k \varrho)^{4}}+O\left([k \varrho]^{-2}\right)\right] \boldsymbol{e}_{\varrho}, \quad k \varrho \ll 1,
$$

which depends on the inverse interparticle distance to the fourth power. Furthermore, the interaction force is antisymmetric $\boldsymbol{F}_{\text {int }}^{\mathrm{rad}}\left(\boldsymbol{r}_{p} \mid \boldsymbol{r}_{s}\right)=-\boldsymbol{F}_{\text {int }}^{\mathrm{rad}}\left(\boldsymbol{r}_{s} \mid \boldsymbol{r}_{p}\right)$. It is worth noticing that the dependence on the inverse interparticle distance $\varrho^{-4}$ has also been found in the acoustic interaction force between two rigid small particles derived by Weiser et al. [31]. In the long-range limit $k \varrho \gg 1$, the acoustic interaction force in the transverse plane is

$$
\boldsymbol{F}_{\text {int }}^{\mathrm{rad}}(\varrho)=-k U_{0}\left\{\frac{\sin (k \varrho)}{k \varrho}+O\left([k \varrho]^{-2}\right)\right\}, \quad k \varrho \gg 1 .
$$


This result has been previously obtained by Zhuk for the interaction of two rigid small particles [21]. Note that the acoustic interaction force decays with the interparticle distance, but that it oscillates in space with two consecutive zeros separated by a half wavelength of the external traveling plane wave. Finally, it should be noted that the short-range interaction depends on the product of the density contrast factors $f_{1, p} f_{1, s}$, whereas in the long-range interaction the dependence is upon the product of the compressibility contrast factors $f_{0, p} f_{0, s}$.

\section{B. Standing plane wave}

Now, consider the case where the external incident wave is a standing plane wave defined by the potential

$$
\phi_{\text {ext }}(z)=\frac{v_{0}}{k} \sin [k(z-h)],
$$

where $h$ is the distance from the first wave node to the origin of the coordinate system. A particle exposed to such a wave will be collected in the potential node if the scattering coefficients satisfy $2 f_{0, p}<-3 f_{1, p}$ and in the potential antinode if $2 f_{0, p}>-3 f_{1, p}$.

We calculate the interaction potential energy by inserting Eqs. (6) and (18) into Eq. (11). Accordingly, we obtain

$$
\begin{aligned}
U(r, \theta)= & \pi E_{0} k^{3} a_{p}^{3} a_{s}^{3}\left(\operatorname { c o s } [ k ( r \operatorname { c o s } \theta - h ) ] \frac { f _ { 1 , p } } { 2 } \left\{f_{1, s} \cos (k h)(1+3 \cos 2 \theta) \frac{\cos (k r)}{(k r)^{3}}+\left[\frac{4}{3} f_{0, s} \sin (k h) \cos \theta \cos k r\right.\right.\right. \\
& \left.\left.+f_{1, s} \cos (k h)(1+3 \cos 2 \theta) \sin (k r)\right] \frac{1}{(k r)^{2}}-\left[f_{1, s} \cos (k h)(1+\cos 2 \theta) \cos (k r)-\frac{1}{3} f_{0, s} \sin (k h) \cos \theta \sin (k r)\right] \frac{1}{k r}\right\} \\
& \left.+\sin [k(r \cos \theta-h)] \frac{2 f_{0, p}}{3}\left\{f_{1, s} \cos (k h) \cos \theta \frac{\cos k r}{(k r)^{2}}+\left[\frac{2}{3} f_{0, s} \sin (k h) \cos (k r)+f_{1, s} \cos (k h) \cos \theta \sin (k r)\right] \frac{1}{k r}\right\}\right) .
\end{aligned}
$$

Using a similar analysis as performed in Sec. III A, we first study the acoustic interaction force between two air bubbles. The force is given by the negative gradient of Eq. (19) considering only the term containing $f_{0, p} f_{0, s}$, and we arrive at the secondary Bjerknes force in a standing plane wave,

$$
\boldsymbol{F}_{\text {int }}^{\mathrm{rad}}(r) \approx-\frac{4 \pi E_{0} k^{2} a_{p}^{3} a_{s}^{3} \kappa_{p} \kappa_{s}}{9 \kappa_{0}^{2} r^{2}} \sin ^{2}(k h) \boldsymbol{e}_{r}, \quad k r \ll 1 .
$$

This is equivalent to the result obtained by Zheng and Apfel [24].

Next we focus on the acoustic interaction force between particles in the transverse plane defined by $\theta=\pi / 2$. In this special case Eq. (19) reduces to

$$
U(\varrho)=U_{0} \sin ^{2}(k h) n_{0}(k \varrho)+U_{1} \cos ^{2}(k h) \frac{n_{1}(k \varrho)}{k \varrho} .
$$

We note that this interaction potential only depends on distance between the source and the probe, and consequently, the acoustic interaction force between particles in the transverse plane is antisymmetric with respect to the probe and the source particles.

According to whether a given set of particles is collected in either the nodal or the antinodal planes of the standing wave, we can choose to let the transverse plane coincide with a nodal plane by setting $k h=0$, in which case all $\sin (k h)$ terms vanish in Eq. (21), and with an antinodal plane by $k h=\pi / 2$, in which case all $\cos (k h)$ terms vanish.

Thus, from the gradient of $U$ in Eq. (21) we obtain the acoustic interaction force between particles in the nodal plane $(k h=0)$ in the short-range limit $k \varrho \ll 1$ to be

$$
\boldsymbol{F}_{\text {int }}^{\mathrm{rad}}(\varrho)=-k U_{1}\left\{\frac{3}{(k \varrho)^{4}}+O\left([k \varrho]^{-2}\right)\right\} \boldsymbol{e}_{\varrho},
$$

which depends on inverse of the interparticle distance to the fourth power. Furthermore, only the density scattering factors and not the compressibility factors enter. In the long-range limit $k \varrho \gg 1$ for the nodal plane, the acoustic interaction force is

$$
\boldsymbol{F}_{\text {int }}^{\mathrm{rad}}(\varrho)=k U_{1}\left\{\frac{2 \cos (k \varrho)}{(k \varrho)^{2}}+O\left([k \varrho]^{-3}\right)\right\} \boldsymbol{e}_{\varrho},
$$

which has an oscillatory behavior with half an external wavelength distance between two consecutive zeros, while it decays with the inverse square of the interparticle distance. Note that only on the density scattering factors appear.

Similarly, in the antinodal plane $(k h=\pi / 2)$, the shortrange limit $k \varrho \ll 1$ of the acoustic interaction force is

$$
\boldsymbol{F}_{\text {int }}^{\mathrm{rad}}(\varrho)=-k U_{0}\left[\frac{1}{(k \varrho)^{2}}+O(1)\right] \boldsymbol{e}_{\varrho},
$$

while the long-distance limit $k \varrho \gg 1$ is

$$
\boldsymbol{F}_{\text {int }}^{\mathrm{rad}}(\varrho)=-k U_{0}\left\{\frac{\sin (k \varrho)}{k \varrho}+O\left([k \varrho]^{-2}\right)\right\} \boldsymbol{e}_{\varrho} .
$$

In the antinodal plane only the compressibility scattering factors occur.

\section{MEAN-FIELD APPROXIMATION}

Going beyond the simple two-particle problem, we now derive an analytical expression for the acoustic interaction force between a probe particle and the particles surrounding it in a homogeneous particle suspension. In Sec. II C we considered $N$ particles with positions $\boldsymbol{r}_{s}$ in a given configuration $\mathcal{S}$ in a suspension of volume $V$. Using Dirac's $\delta$ function $\delta(\boldsymbol{r})$, we can formally rewrite the $\operatorname{sum} \mathcal{U}$ over pair potentials $U$ as an 
integral,

$$
\begin{aligned}
\mathcal{U}\left(\boldsymbol{r}_{p}\right) & =\sum_{\boldsymbol{r}_{s} \in \mathcal{S}}^{\prime} U\left(\boldsymbol{r}_{p} \mid \boldsymbol{r}_{s}\right)=\int_{V} U\left(\boldsymbol{r}_{p} \mid \boldsymbol{r}\right) n(\boldsymbol{r}) d \boldsymbol{r}, \\
n(\boldsymbol{r}) & =\sum_{\boldsymbol{r}_{s} \in \mathcal{S}}^{\prime} \delta\left(\boldsymbol{r}-\boldsymbol{r}_{s}\right),
\end{aligned}
$$

where $n(\boldsymbol{r})$ can be interpreted as the particle concentration field. In a mean-field approximation, $n(\boldsymbol{r})$ is smoothened, such that the number $d N$ of particles particles in a small volume $d \boldsymbol{r}$ at any given position $\boldsymbol{r}$ is given by $d N=n(\boldsymbol{r}) d \boldsymbol{r}$. For a homogeneous suspension, we have $n(\boldsymbol{r}) \approx N / V$, and the interaction potential experienced by the probe particle is well approximated by

$$
\mathcal{U}\left(\boldsymbol{r}_{p}\right) \approx \frac{N}{V} \int_{V} U\left(\boldsymbol{r}_{p} \mid \boldsymbol{r}\right) d \boldsymbol{r} .
$$

This mean-field approximation is expected to improve for an increasing number of source particles per volume.

To illustrate the mean-field approximation in the acoustic interaction force problem, we assume that the source particles are uniformly distributed within a circular region of radius $R$ and thickness $2 a_{s}$ at the antinodal plane (the $x y$ plane) of the external standing plane wave Eq. (18). The volume occupied by the particle distribution is thus $V=2 \pi R^{2} a_{s}$. The probe particle is placed at the origin of the coordinate system, while the center of the disk-shaped source-particle region is displaced backwards along what is defined to be the $x$ axis to the position $-r_{p} \boldsymbol{e}_{x}$. With this configuration and using Eq. (21), the pair-interaction potential $U\left(\boldsymbol{r}_{p} \mid \boldsymbol{r}_{s}\right)$ becomes

$$
U\left(\boldsymbol{r}_{p} \mid \boldsymbol{r}_{s}\right)=-2 U_{0} \frac{\cos k r_{s}}{k r_{s}}, \text { with } \boldsymbol{r}_{p}=\mathbf{0},
$$

while the limits of the integration region $V$ in the expression (24b) for the total interaction potential requires some analysis. Using the cylindrical polar coordinates $(r, \varphi, z)$ for the source position $\boldsymbol{r}_{s}$, we find that in the direction $\varphi$, a source particle can at most be at the distance $R^{\prime}(\varphi)$ from the probe particle,

$$
R^{\prime}(\varphi)=\sqrt{R^{2}-x_{p}^{2} \sin ^{2} \varphi}-r_{p} \cos \varphi .
$$

The total interaction potential $\mathcal{U}$ having a strength of $\mathcal{U}_{0}=$ $2 N U_{0} / \pi$ for the probe particle at $\boldsymbol{r}_{p}=\mathbf{0}$, becomes

$$
\begin{aligned}
\mathcal{U}\left(\boldsymbol{r}_{p}\right) & =-\frac{2 N U_{0}}{\pi R^{2}\left(2 a_{s}\right)} \int_{0}^{2 \pi} d \varphi \int_{-a_{s}}^{a_{s}} d z \int_{0}^{R^{\prime}(\varphi)} d r r \frac{\cos (k r)}{k r} \\
& =-\mathcal{U}_{0} \int_{0}^{2 \pi} d \varphi \sin \left[k R^{\prime}(\varphi)\right], \quad \mathcal{U}_{0}=U_{0} \frac{2 N}{\pi}
\end{aligned}
$$

For an arbitrary position $\boldsymbol{r}_{p}$ of the probe particle relative to the center of the source-particle region, this integral can be evaluated numerically. However, for small displacements $r_{p} \ll R$, we can obtain an analytical expression by Taylor
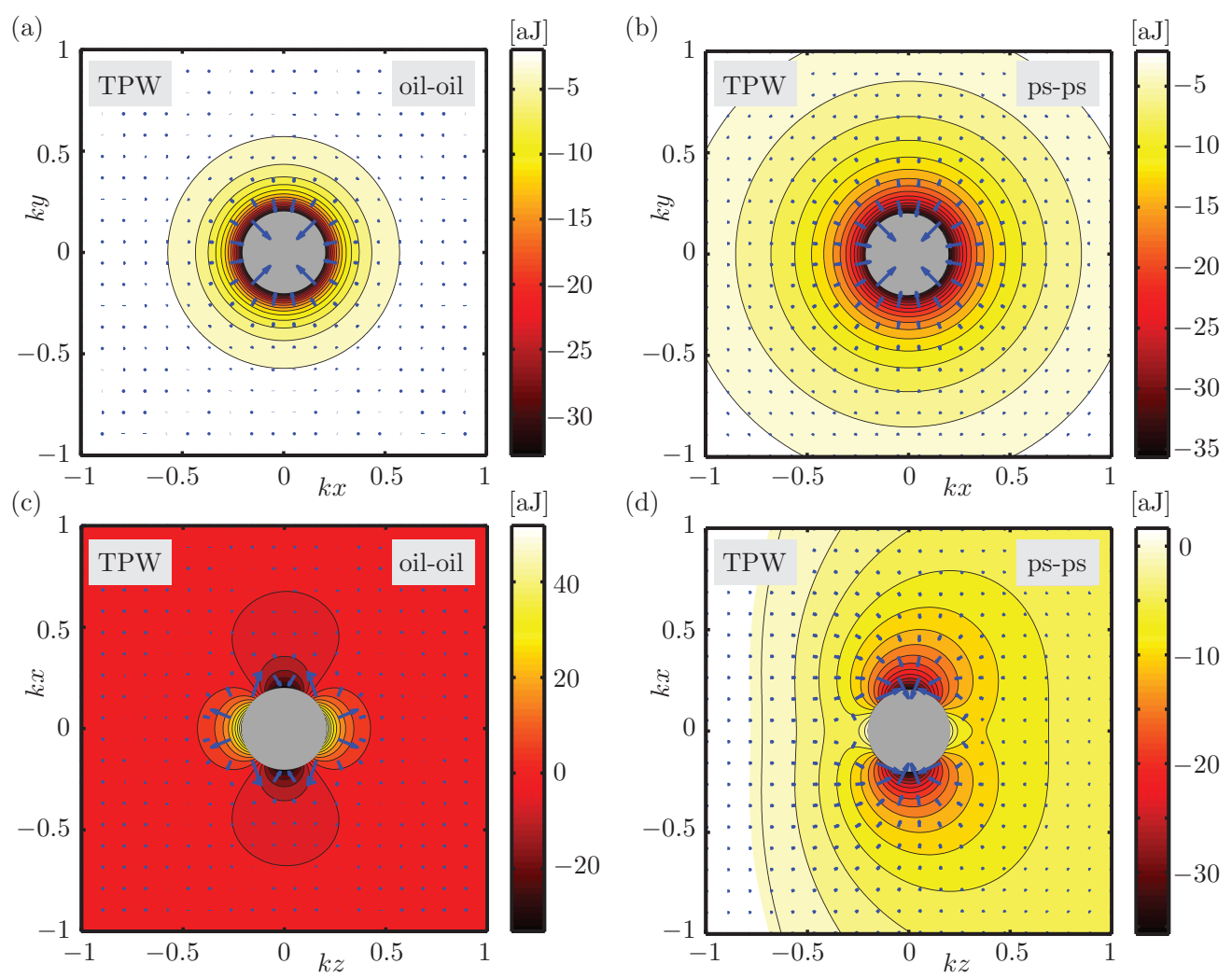

FIG. 2. (Color online) The acoustic interaction pair potential $U\left(\boldsymbol{r}_{p} \mid \mathbf{0}\right)$ [Eq. (14), contours] and force $\boldsymbol{F}_{\text {int }}^{\text {rad }}\left(\boldsymbol{r}_{p} \mid \mathbf{0}\right)=-\nabla U$ (arrows) between a pair of identical $12-\mu \mathrm{m}$ particles induced by the traveling plane wave (TPW) Eq. (13), with the source particle located at the origin, $\boldsymbol{r}_{\mathrm{s}}=\mathbf{0}$, for $k r>0.2$. (a) Silicone oil droplets (oil-oil), with the probe $\boldsymbol{r}_{p}=(x, y, 0)$ in the transverse $x y$ plane. (b) Same as (a) but for polystyrene microparticles (ps-ps). (c) Same as (a), but with the probe $\boldsymbol{r}_{p}=(x, 0, z)$ in the parallel $x z$ plane (oil-oil). (d) Same as (b), but with the probe $\boldsymbol{r}_{p}=(x, 0, z)$ in the parallel $x z$ plane (ps-ps). 
expanding the integrand,

$$
\begin{aligned}
\sin \left[k R^{\prime}(\varphi)\right] \approx & \sin k R-k r_{p} \cos \varphi \cos k R \\
& -\frac{\sin ^{2} \varphi \cos k R+k R \cos ^{2} \varphi \sin k R}{2 k R}\left(k r_{p}\right)^{2},
\end{aligned}
$$

which upon insertion into Eq. (28) leads to

$$
\begin{aligned}
\mathcal{U}\left(\boldsymbol{r}_{p}\right)= & -\mathcal{U}_{0}\left\{\frac{\sin k R}{(k R)^{2}}-\left[\frac{\sin k R}{(k R)^{2}}+\frac{\cos k R}{(k R)^{3}}\right] \frac{\left(k r_{p}\right)^{2}}{4}\right\} \\
& +O\left[\left(k r_{p}\right)^{4}\right] .
\end{aligned}
$$

By taking the negative gradient $-\nabla_{p}=-\boldsymbol{e}_{r} \partial / \partial_{r_{p}}$ relative to the probe position, we determine the acoustic interaction force on the probe particle to be

$$
\boldsymbol{F}_{\text {rad }}^{\text {int }}\left(\boldsymbol{r}_{p}\right)=-\frac{k \mathcal{U}_{0}}{2}\left[\frac{\sin k R}{(k R)^{2}}+\frac{\cos k R}{(k R)^{3}}\right] k \boldsymbol{r}_{p}+O\left[\left(k r_{p}\right)^{3}\right] .
$$

We note that, as expected, the interaction force is zero in the case $r_{p}=0$, where the source particles are symmetrically distributed around the probe particle. Moreover, the interaction force tends to zero in the limit $k R \gg 1$ for fixed $k r_{p}$, a fact that can be explained by the decreasing degree of asymmetry in the source particles characterized by the decreasing ratio $r_{p} / R$.
We also note that if the sign of the compressibility factors $f_{0, p}$ and $f_{0, s}$ are the same, the symmetric position $r_{p}=0$ is a stable equilibrium point if $\cos k R+k R \sin k R>0$, in which case the particles will be attracted to the center of the source region. Finally, we note that the frequency dependence of the interaction force is governed by the trigonometric factors. In the case of a small disk region, $k R \ll 1$, we have that $(\cos k R) /(k R)^{3} \approx(k R)^{-3}$ dominates. Consequently, in this case the acoustic interaction force depends on the wave number as $k^{2}$ and thus quadratically with frequency.

\section{RESULTS AND DISCUSSION}

In this section, based on direct numerical evaluations of $U(\boldsymbol{r} \mid \mathbf{0})$ in Eqs. (14) and (19) for a traveling and standing place wave, respectively, we calculate the acoustic interaction force between a pair of silicone oil droplets and a pair of polystyrene microparticles suspended in water at room temperature. The water is characterized by its density $\rho_{0}=1000 \mathrm{~kg} / \mathrm{m}^{3}$ and speed of sound $c_{0}=1500 \mathrm{~m} / \mathrm{s}$. Using the material parameters given in Ref. [32], the scattering factors $f_{0}$ and $f_{1}$ given in Eq. (4) are found to be $\left(f_{0}, f_{1}\right)=(-0.08,0.07)$ for silicone oil and $\left(f_{0}, f_{1}\right)=(0.46,0.038)$ for polystyrene. For the external wave, we choose the following typical parameter values from actual acoustophoresis experiments [33]: frequency $\omega /(2 \pi)=$ $2 \mathrm{MHz}$, wave number $k=8378 \mathrm{~m}^{-1}$, and energy density $E_{0}=10 \mathrm{~J} / \mathrm{m}^{3}$. The microparticle radius is $a_{p}=a_{s}=12 \mu \mathrm{m}$,
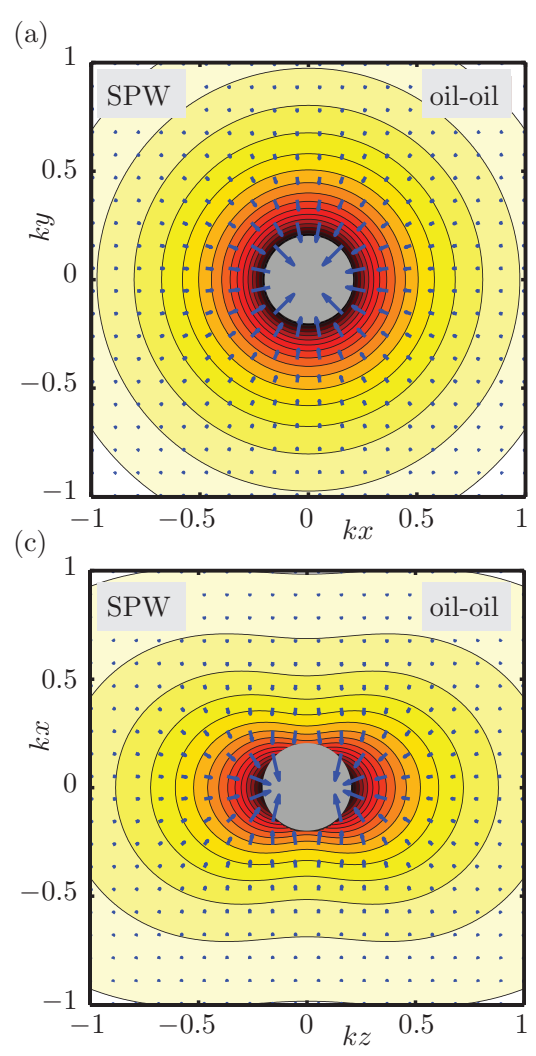

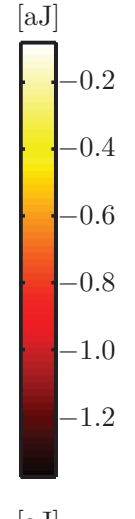

(b)
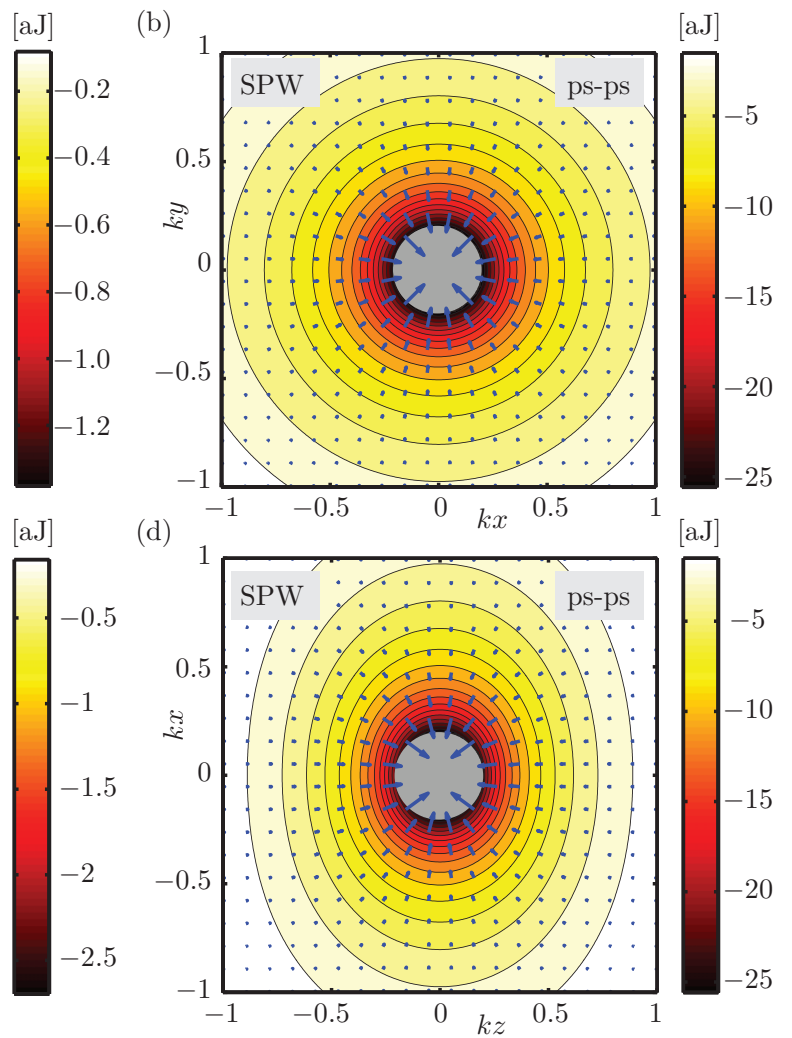

FIG. 3. (Color online) The acoustic interaction pair potential $U\left(\boldsymbol{r}_{p} \mid \mathbf{0}\right)$ [Eq. (19), contours] and force $\boldsymbol{F}_{\text {int }}^{\mathrm{rad}}\left(\boldsymbol{r}_{p} \mid \mathbf{0}\right)=-\nabla U$ (arrows) between a pair of identical $12-\mu \mathrm{m}$ particles induced by the standing plane wave (SPW) Eq. (18), with $k h=\pi / 2$ and the source particle located at the origin, $\boldsymbol{r}_{\mathrm{s}}=\mathbf{0}$ for $k r>0.2$. (a) Silicone oil droplets (oil-oil), with the probe $\boldsymbol{r}_{p}=(x, y, 0)$ in the transverse $x y$ plane. (b) Same as (a) but for polystyrene microparticles (ps-ps). (c) Same as (a), but with the probe $\boldsymbol{r}_{p}=(x, 0, z)$ in the parallel $x z$ plane (oil-oil). (d) Same as (b), but with the probe $\boldsymbol{r}_{p}=(x, 0, z)$ in the parallel $x z$ plane (ps-ps). 
so we obtain $k a_{s}=k a_{p}=0.1$. Below, the source particle is positioned at $\boldsymbol{r}_{\mathbf{s}}=\mathbf{0}$, whereas the probe is placed at any position in space, $\boldsymbol{r}_{p}=\boldsymbol{r}$.

We compute the acoustic interaction force $\boldsymbol{F}_{\text {int }}^{\text {rad }}$ due to an external traveling wave or a standing wave plane, from the pair potential $U(\boldsymbol{r} \mid \mathbf{0})$ in Eqs. (14) and (19), respectively, as $\boldsymbol{F}_{\text {int }}^{\mathrm{rad}}=$ $-\nabla U(\boldsymbol{r} \mid \mathbf{0})$ using Mathematica software [34]. The probe position $\boldsymbol{r}_{p}$ is presented in the scaled Cartesian coordinates $k \boldsymbol{r}_{p}=(k x, k y, k z)$.

\section{A. Particle pairs in a traveling plane wave}

In Fig. 2, we show the pair potential $U(\boldsymbol{r} \mid \mathbf{0})$ (contour plot) and the corresponding acoustic interaction force $\boldsymbol{F}_{\text {int }}^{\mathrm{rad}}$ (arrows) induced by the external traveling plane wave Eq. (13) propagating along the $z$ axis for a pair of oil microdroplets and a pair of polystyrene microparticles, respectively. In the (transverse) $x y$ plane Figs. 2(a) and 2(b), the acoustic interaction force is central and also attractive. The force is central because the microparticles directly interact through each other's scattered wave, while the external wave amplitude is the same for both microparticles. Note that in the short-range distance, the acoustic interaction force is about $-U / a_{p}$. Hence, the force magnitude on the oil and the polystyrene probe microparticles is about $1 \mathrm{pN}$. In the (parallel) $x z$ plane Figs. 2(c) and 2(d), the situation is different. The acoustic interaction force is not a central force, because the amplitude of the external wave is not the same for the microparticles. In the short-range distance, we observe a repulsive region in the vicinity of the $z$ axis, whereas an attractive region is seen close to the $x$ axis. The interaction force magnitude is about $1 \mathrm{pN}$ for both oil and polystyrene microparticles.

\section{B. Particle pairs in a standing plane wave}

In Fig. 3, we show the pair potential $U(\boldsymbol{r} \mid \mathbf{0})$ (contour plot) and the corresponding acoustic interaction force $\boldsymbol{F}_{\text {int }}^{\text {rad }}$ (arrows) induced by the external standing plane wave, Eq. (18), along the $z$ axis. We set $k h=\pi / 2$ resulting in an antinode in the transverse $x y$ plane for a pair of oil microdroplets and a pair of polystyrene microparticles, respectively. For both particle pairs, the primary radiation force focus particles in the antinodal plane as discussed in Sec. III B. In the transverse $x y$ plane [Figs. 3(a) and 3(b)], the acoustic interaction force is central and attractive for both oil and polystyrene microparticles. We note that the interaction force between two polystyrene microparticles is stronger than that for oil microdroplets. In the parallel plane [Figs. 3(c) and 3(d)], the interaction force is not central but is almost attractive in the vicinity of the source microparticle. The magnitude of $\boldsymbol{F}_{\text {int }}^{\mathrm{rad}}$ between the oil and the polystyrene probe microparticles is nearly $1.0 \mathrm{pN}$.
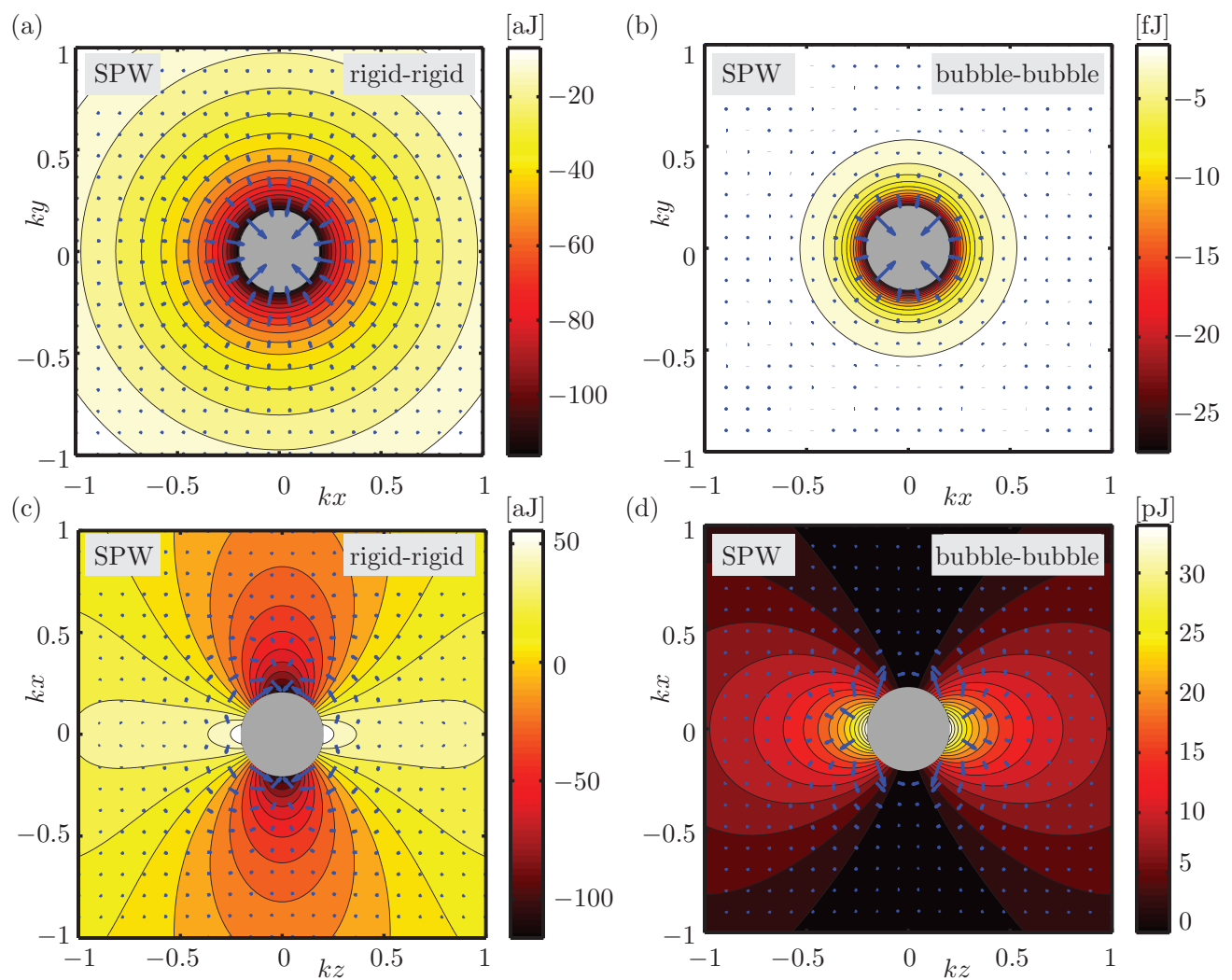

FIG. 4. (Color online) The acoustic interaction pair potential $U\left(\boldsymbol{r}_{p} \mid \mathbf{0}\right)$ [Eq. (19), contours] and force $\boldsymbol{F}_{\text {int }}^{\text {rad }}\left(\boldsymbol{r}_{p} \mid \mathbf{0}\right)=-\nabla U$ (arrows) between a pair of identical $12-\mu \mathrm{m}$ particles induced by the standing plane wave (SPW) Eq. (18), with the source particle located at the origin, $\boldsymbol{r}_{\mathrm{s}}=\mathbf{0}$ for $k r>0.2$. (a) Rigid microparticles (rigid-rigid), with $f_{0, p}=f_{0, s}=f_{1, p}=f_{1, s}=1, k h=\pi / 2$ (antinode), and the probe $\boldsymbol{r}_{p}=(x, y, 0)$ in the transverse $x y$ plane. (b) Same as (a) but for microbubbles (bubble-bubble) with $f_{0, p}=f_{0, s} \approx-10^{5}, f_{1, p}=f_{1, s} \approx-2$, and $k h=0$ (node). (c) Same as (a), but with the probe $\boldsymbol{r}_{p}=(x, 0, z)$ in the parallel $x z$ plane (rigid-rigid). (d) Same as (b), but with the probe $\boldsymbol{r}_{p}=(x, 0, z)$ in the parallel $x z$ plane (bubble-bubble). 
To emphasize the importance of treating the particles with their correct compressibilities and densities, we present in Fig. 4 the pair potential $U(\boldsymbol{r} \mid \mathbf{0})$ (contour plot) and the corresponding acoustic interaction force $\boldsymbol{F}_{\text {int }}^{\text {rad }}$ (arrows) induced by the same external standing plane wave as above, but now for the limiting cases for a pair of completely rigid and heavy microparticles $\left(f_{0, s}=f_{0, p}=f_{1, s}=f_{1, p}=1\right)$ and a pair of highly compressible air microbubbles $\left(f_{0, s}=f_{0, p} \approx-10^{5}\right.$ and $f_{1, s}=f_{1, p} \approx-2$ ). The radial oscillation dynamics of the microbubbles is considered in this analysis. The primary radiation force focuses the rigid microparticles and microbubbles at the antinodal and nodal planes, respectively, as discussed in Sec. III B. Thus, we set $k h=\pi / 2$ for the rigid microparticles, while we chose $k h=0$ for the microbubbles. In the transverse $x y$ plane [Figs. 4(a) and 4(b)], the acoustic interaction force is central and attractive for both rigid microparticles and microbubbles. The interaction force between two microbubbles is much stronger (with magnitude of about $0.1 \mathrm{nN}$ ) than that for any other microparticle considered in this study. In the parallel plane [Figs. 4(c) and 4(d)], the interaction force is not central and has a similar pattern for both microparticles (rigid and bubble) analyzed here. The interaction force drives the probe microparticle away from the standing wave axial line $(z$ axis) toward the transverse line ( $x$ axis). Moreover, the probe microparticles tend to collect on the poles of the spherical region $k r<0.2$ along the $x$ axis. Summarizing the four particle cases, we note that the largest acoustic pair-interaction potential is experienced by the air bubbles $(\simeq 30 \mathrm{pJ})$, followed in decreasing order by rigid particles $(\simeq 100 \mathrm{aJ})$, polystyrene particles $(\simeq 20 \mathrm{aJ})$, and oil droplets $(\simeq 2 \mathrm{aJ})$. Also the angular dependence varies with the particle parameters, most clearly in the parallel $x z$ plane, where a potential minimum is along the $z$ axis for the oil-oil case [Fig. 3(c)], but along the $x$ axis for the other three cases [Figs. 3(d), 4(c), and 4(d)], the minimum region being most extended for the bubble-bubble case [Fig. 4(d)].

Finally, for the same standing plane wave, we present in Fig. 5 the acoustic interaction force between two different particles, a polystyrene source and a silicone oil probe. In the transverse $x y$ plane [Fig. 5(a)], the acoustic interaction force in short-range distances is central and repulsive. The radiation force magnitude is less than $1 \mathrm{pN}$. In the parallel $x z$ plane [Fig. 5(b)], the main role of the acoustic interaction force is also to be repulsive.

\section{Emulsion of oil droplets in water}

As a last numerical example, we consider the multiparticle system comprising an emulsion of soybean oil droplets of radius $a_{s}=12 \mu \mathrm{m}$ in water. The scattering factors are $f_{0, s}=$ -0.11 and $f_{1, s}=-0.06$ [35], so according to Sec. III B, the oil microdroplets will collect in a node when exposed to a standing plane wave. We therefore study the effects of such a wave described by Eq. (18) with $k h=0$ droplets initially uniformly distributed in the transverse (nodal) $x y$ plane in a circular disk-shaped region of radius $R=5 \mathrm{~mm}$ and thickness $2 a_{s}$. The mean interdroplet distance is assumed to be $10 a=$ $120 \mu \mathrm{m}$, which corresponds to $N \approx 3800$ droplets within the disk-shaped region. The interaction potential is then calculated by numerical integration of the mean-field approximation
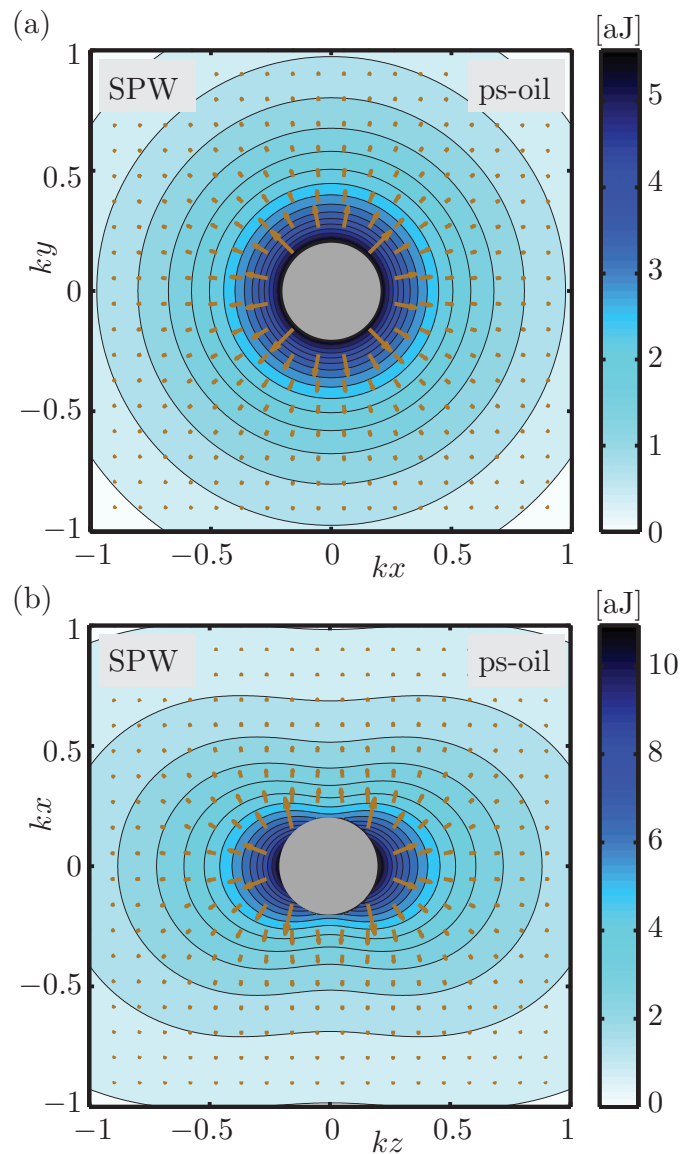

FIG. 5. (Color online) Same as Figs. 3(a) and 3(c), but with two different particles (oil-ps): a $12-\mu \mathrm{m}$ silicone oil droplet as the probe particle at $\boldsymbol{r}_{p}=\boldsymbol{r}$ and a $12-\mu \mathrm{m}$ polystyrene particle as the source particle at $\boldsymbol{r}=\mathbf{0}$.

(28) for $\mathcal{U}$. Subsequently, the acoustic interaction force was determined by calculating numerically the negative gradient of this $\mathcal{U}$. In this case the pair-interaction potential has the strength has the value $U_{0}=8.1 \times 10^{-20} \mathrm{~J}$, yielding a total interaction strength of $\mathcal{U}_{0}=0.2 \mathrm{fJ}$. Hence, the magnitude of the acoustic interaction force in the short-range distance is about $\mathcal{U}_{0} / a_{s}=16 \mathrm{pN}$.

In Fig. 6, we depict the normalized interaction potential energy $\tilde{\mathcal{U}}=\mathcal{U} / \mathcal{U}_{0}$ (contour plot) and the associated acoustic interaction force $\boldsymbol{F}_{\text {int }}^{\text {rad }}$ (arrows) on a probe droplet placed in the transverse $x y$ plane in the disk-shaped region of source droplets. The potential $\tilde{\mathcal{U}}$ exhibits concentric local maxima and minima, with the global maximum localized at $k r=0$. Hence, microdroplets have the tendency to move away from the central region. This is in agreement with Eq. (31), because here $(\cos k R+k R \sin k R) \approx-36$. On the other hand, the potential minima will attract the nearby oil microdroplets. Therefore, microdroplets may aggregate in the minima concentric regions throughout the emulsion. Note that the distance between two consecutive minima is about $10 \%$ of the incident wavelength. Furthermore, the magnitude of the acoustic interaction force is less than $0.02 \mathrm{nN}$. 


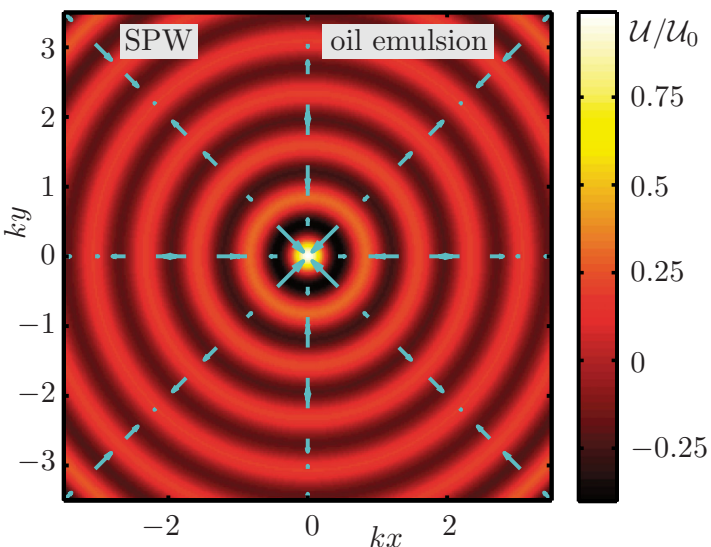

FIG. 6. (Color online) The normalized interaction potential $\mathcal{U} / \mathcal{U}_{0}$ (contour plot) of the acoustic interaction force $\boldsymbol{F}_{\text {int }}^{\text {rad }}$ (arrows) in an aqueous emulsion of soybean oil droplets of radius $a_{s}=12 \mu \mathrm{m}$ in the external standing plane wave (SPW) defined in Eq. (18). The emulsion consists of $N \approx 3800$ droplets in a cylindrical disk region of radius $R=5 \mathrm{~mm}$ and thickness $2 a_{s}$ at the nodal $x y$ plane. Here $\mathcal{U}_{0}=0.2 \mathrm{fJ}$.

\section{SUMMARY AND CONCLUSION}

We have developed a potential theory for the acoustic interaction forces in a collection of $N$ suspended microparticles in an ideal fluid, considering the long-wavelength limit $a_{s} \ll \lambda$ $(s=1,2, \ldots, N)$. The microparticles were considered to be either compressible liquid droplets, air microbubbles, elastic solid spheres, or heavy rigid microspheres.
In our analysis, the acoustic interaction force between two particles is expressed in terms of the negative gradient of the pair-interaction potential energy function. In turn, this function depends on the product of the external and scattering velocity potentials. We have shown that the multiscattering contribution to the acoustic interaction force on a particle placed at $\boldsymbol{r}_{p}$ is dominated by scattering waves having undergone only a single prior scattering event due to a source particle located at $\boldsymbol{r}_{s}$ $(s \neq p)$.

The investigations of the interparticle interactions under a traveling and a standing plane wave have shown that the acoustic interaction forces might be attractive or repulsive for short-range interaction. In the transverse $x y$ plane to the wave propagation direction, the acoustic force is a central force, while in in the parallel $x z$ plane this does not happen.

We have also presented a mean-field theory based on the continuous limit of the acoustic interaction potential energy for an emulsion formed by oil droplets in water. Under a standing plane wave, oil droplets have the tendency to cluster in concentric regions on the transverse $x y$ plane.

The theoretical predictions discussed in this work might be confirmed in microparticle acoustophoresis experiments using methods such as optical trapping [36], 3D astigmatism particle tracking velocimetry [4], microparticle image velocimetry [37], and ultrasonic demulsification [38].

\section{ACKNOWLEDGMENTS}

This work was supported by CAPES, Ministry of Education in Brazil, Grant No. 17997-12-7, and by the Danish Council for Independent Research (DFF), Technology and Production Sciences, Grant No. 11-10702.
[1] T. Laurell, F. Petersson, and A. Nilsson, Chem. Soc. Rev. 36, 492 (2007).

[2] H. Bruus, J. Dual, J. Hawkes, M. Hill, T. Laurell, J. Nilsson, S. Radel, S. Sadhal, and M. Wiklund, Lab Chip 11, 3579 (2011).

[3] X. Ding, S.-C. S. Lin, B. Kiraly, H. Yue, S. Li, I.-K. Chiang, J. Shi, S. J. Benkovic, and T. J. Huang, Proc. Natl. Acad. Sci. USA 109, 11105 (2012).

[4] P. B. Muller, M. Rossi, Á. G. Marín, R. Barnkob, P. Augustsson, T. Laurell, C. J. Kähler, and H. Bruus, Phys. Rev. E 88, 023006 (2013).

[5] R. Barnkob, P. Augustsson, T. Laurell, and H. Bruus, Phys. Rev. E 86, 056307 (2012).

[6] A. A. Doinikov, J. Acoust. Soc. Am. 101, 722 (1997).

[7] S. D. Danilov and M. A. Mironov, J. Acoust. Soc. Am. 107, 143 (2000).

[8] M. Settnes and H. Bruus, Phys. Rev. E 85, 016327 (2012).

[9] G. T. Silva, J. Acoust. Soc. Am. 136, 2405 (2014).

[10] L. V. King, Proc. R. Soc. London, Ser. A 147, 212 (1934).

[11] K. Yosioka and Y. Kawasima, Acustica 5, 167 (1955).

[12] L. P. Gor'kov, Doklady Akademii Nauk SSSR 140, 88 (1961) [Sov. Phys.-Dokl. 6, 773 (1962)].

[13] V. F. K. Bjerknes, Fields of Force (Columbia University, New York, 1906).

[14] W. König, Ann. Phys. 278, 549 (1891) [in German].

[15] T. F. W. Emblenton, J. Acoust. Soc. Am. 34, 1714 (1962).
[16] W. L. Nyborg, Ultrasound Med. Biol. 15, 93 (1989).

[17] A. A. Doinikov and S. T. Zavtrak, Phys. Fluids 7, 1923 (1995).

[18] L. Crum, J. Acoust. Soc. Am. 57, 1363 (1975).

[19] A. A. Doinikov and S. T. Zavtrak, Ultrasonics 34, 807 (1996).

[20] A. A. Doinikov, J. Acoust. Soc. Am. 99, 3373 (1996).

[21] A. P. Zhuk, Sov. Appl. Mech. 21, 307 (1985) [in English].

[22] A. A. Doinikov, J. Acoust. Soc. Am. 106, 3305 (1999).

[23] A. A. Doinikov, J. Acoust. Soc. Am. 111, 1602 (2002).

[24] X. Zheng and R. E. Apfel, J. Acoust. Soc. Am. 97, 2218 (1995).

[25] A. A. Doinikov, J. Fluid Mech. 444, 1 (2001).

[26] P. M. Morse and K. U. Ingard, Theoretical Acoustics (Princeton University Press, Princeton, NJ, 1986).

[27] A. D. Pierce, Acoustics (Acoustical Society of America, Melville, NY, 1989).

[28] D. T. Blackstock, Physical Acoustics (Wiley \& Sons, Hoboken NJ, 2000).

[29] L. D. Landau and E. M. Lifshitz, Fluid Mechanics, 2nd ed. (Pergamon Press, Oxford, 1993), Vol. 6.

[30] H. Überall, Handbook of Acoustics (Wiley \& Sons, New York, 1998), Chap. 4, pp. 47-60.

[31] M. A. H. Weiser, R. E. Apfel, and E. A. Neppiras, Acta Acust. Acust. 56, 114 (1984).

[32] G. S. Kino, Acoustic Waves: Devices, Imaging, and Analog Signal Processing (Prentice-Hall, Upper Saddle River, NJ, 1987), p. 548. 
[33] R. Barnkob, P. Augustsson, T. Laurell, and H. Bruus, Lab Chip 10, 563 (2010).

[34] Wolfram Research Inc., Mathematica 8.0 (Wolfram Research Inc., Champaign, IL, 2010).

[35] J. N. Coupland and D. J. McClements, J. Am. Oil Chem. Soc. 74, 1559 (1997).
[36] S. Lakämper, A. Lamprecht, I. A. T. Schaap, and J. Dual, Lab Chip 15, 290 (2015).

[37] P. Augustsson, R. Barnkob, S. T. Wereley, H. Bruus, and T. Laurell, Lab Chip 11, 4152 (2011).

[38] S. Nii, S. Kikumoto, and H. Tokuyama, Ultrasound Sonochem. 16, 145 (2009). 